\title{
Melt Conditioned Direct Chill Casting (MC-DC) of Wrought Al-alloys
}

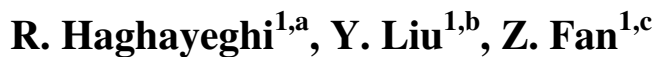 \\ ${ }^{1}$ BCAST, Brunel University, Uxbridge, Middlesex, UB8 3PH, UK \\ ${ }^{\text {a }}$ Reza.Haghayeghi@Brunel.ac.uk, ${ }^{\mathrm{b}}$ Yuan.liu@Brunel.ac.uk, ${ }^{\mathrm{c}}$ Zhongyun.Fan@Brunel.ac.uk
}

\begin{abstract}
Melt Conditioned Direct Chill (MC-DC) casting is a new development for producing high-quality billets and slabs. In the MC-DC process, liquid metal is continuously fed into a MCAST (melt conditioning by advanced shear technology) machine, where the liquid metal is subjected to high shear rate and high degree of turbulence provided by a twin screw mechanism at temperatures either above or below the alloy liquidus, and the conditioned liquid metal is then fed continuously into a Direct Chill (DC) caster to produce billets or slabs. The MC-DC process is applicable to both Aland $\mathrm{Mg}$-alloys. In this paper we present our experimental investigations of the effects of processing parameters on the microstructural and compositional uniformity of 5xxx and 7xxx series Al-alloys. It has been confirmed by our experiments that the MC-DC process can produce billets and slabs with fine and uniform microstructure, uniform chemical compositions and much reduced cast defects, such as porosity and cracks.
\end{abstract}

Keywords: Melt conditioning, direct chill casting, solidification, aluminium alloy.

\section{Introduction}

Aluminium alloy have unique characteristics such as high specific strength and good cast ability that make them attractive for structural applications in automotive, aerospace and many other general engineering fields. Among aluminium alloys wrought products are highly useful due to their specific properties such as high Ultimate Tensile Strength (UTS) and Yield Strength (YS). The current method for producing these alloys starts with direct chill (DC) casting. Most of the research on wrought alloys has been focused on forming in solid state, only limited efforts on improving the casting processes, such as magneto-hydrodynamic stirring (MHD) [1,2] has been done. These processes have their own problems such as complexity, size of ingot and production rate. Recently, BCAST at Brunel University has introduced a new technique, Melt Conditioned Direct Chill (MCDC) casting process [3]. This technique is proven to be more efficient and produces high quality ingots with fine and uniform microstructures and does not have the restrictions of billet size. The MC-DC process combines the well-developed MCAST process [4] for melt conditioning and the conventional direct chill (DC) process for billet or slab production. The MC-DC process exploits the benefit provided by solidification under intensive forced convection. It has been demonstrated that the MC-DC process can offer Mg-alloy billets with fine and uniform microstructure, uniform chemical compositions and much reduced cast defects, such as porosity and cracks. In this paper we report our experimental results on microstructural refinement and compositional uniformity of 5754 and 7075 Al-alloy billets produced by the MC-DC process.

\section{Experimental}

The MC-DC equipment consists of two basic functional units: a MCAST machine for melt conditioning and a standard DC caster for production of billets or slabs, as schematically illustrated in Fig. 1. The MCAST machine has a pair of co-rotating, fully intermeshing and self-wiping screws. The screws have specially designed profiles to achieve high shear rate and high degree of turbulence. In addition, there is a large ever-renewing interfacial area between the melt and the 
screws and the barrel, ensuring an extremely efficient heat transfer. The conditioned liquid metal is then continuously fed into a DC caster for billet production. The main objective of the MC-DC process is to produce a billet or a slab with fine and uniform microstructure with consistent chemical composition. For this work both the standard TP1 mould [5] with a cooling rate of $3.5 \mathrm{~K} / \mathrm{sec}$ and a water-cooled copper mould with $80 \mathrm{~mm}$ inner diameter [3] were used to simulate the DC casting conditions. Liquid metal of 7075 and 5754 was prepared in a resistance furnace using graphite crucible. The furnace temperature was set at $750^{\circ} \mathrm{C}$. The alloy composition is given in Table 1.

Table 1: Chemical composition of 7075 and 5754 aluminium alloys (wt.\%).

\begin{tabular}{|l|l|l|l|l|l|l|l|l|l|}
\hline Alloy & $\mathrm{Zn}$ & $\mathrm{Mg}$ & $\mathrm{Cu}$ & $\mathrm{Cr}$ & $\mathrm{Fe}$ & $\mathrm{Mn}$ & $\mathrm{Ti}$ & $\mathrm{Si}$ & $\mathrm{Al}$ \\
\hline 7075 & 5.56 & 1.95 & 1.64 & 0.19 & 0.10 & 0.03 & 0.01 & 0.07 & Bal. \\
\hline 5754 & 0.06 & 4.0 & 0.05 & 0.11 & 0.25 & 0.25 & 0.01 & 0.08 & Bal. \\
\hline
\end{tabular}

Liquid metal with around $50^{\circ} \mathrm{C}$ super heat was fed into the MCAST machine and sheared at $628^{\circ} \mathrm{C}$ and $650^{\circ} \mathrm{C}$ for 7075 (below liquidus) and 5754 (above liquidus), respectively, for 60 seconds at $800 \mathrm{rpm}$. The conditioned liquid 5754 or slurry 7075 were then fed into the TP1 mould or a watercooled copper mould for producing the solidified samples, respectively. To evaluate the microstructures of the alloys, samples were cut from the specific positions of the solid piece, grounded, polished and anodised at an optimum voltage of $(20-40 \mathrm{~V})$ in a $2 \%$ aqueous solution of tetra flouroboric acid $\left(\mathrm{HBF}_{4}\right)$ to obtain the grain size. The microstructures of samples were examined by an optical microscope and the chemical compositions were measured by an optical mass spectrometer. Also, the effect of grain refiner on DC casting was also investigated because for conventional DC casting, grain refiners are widely used for structural refinement.

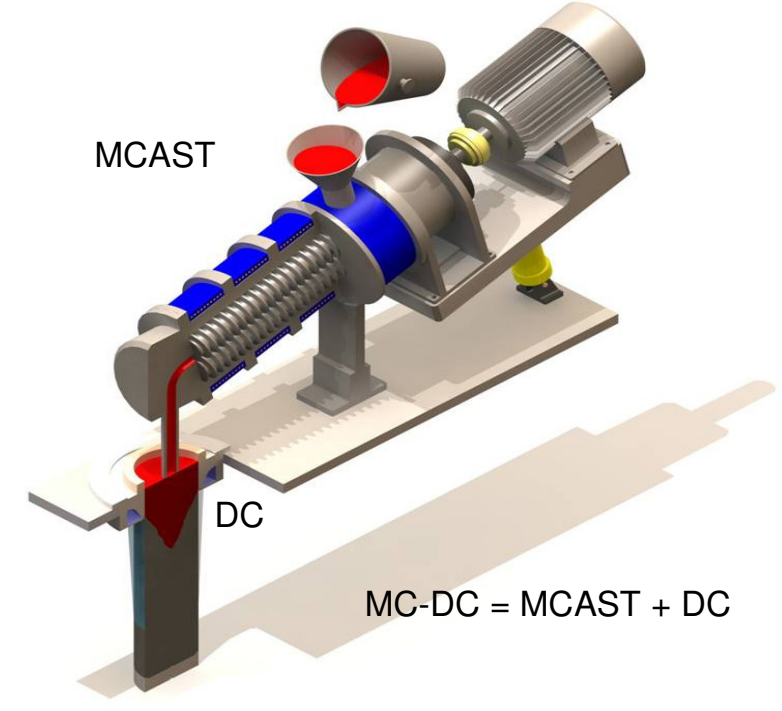

Fig. 1 Schematic illustration of MC-DC process.

The interaction between intensive melt shearing and grain refiner addition was also investigated using 5754Al alloy and Al-5Ti-1B grain refiner.

\section{Results}

The conventional DC cast billet exhibits the typical 3-zone structure, the chilled zone with fine equiaxed grains at the billet surface, and coarse equiaxed structure at the centre of the billet and columnar grains between the chilled zone and coarse equiaxed zone (Figs. $2 \mathrm{c}$ and $2 \mathrm{~d}$ ). However, the MC-DC cast billet has a fine equiaxed grain structure throughout the entire cross section of the billet, as shown in the Figs $2 \mathrm{a}$ and $2 \mathrm{~b}$. 

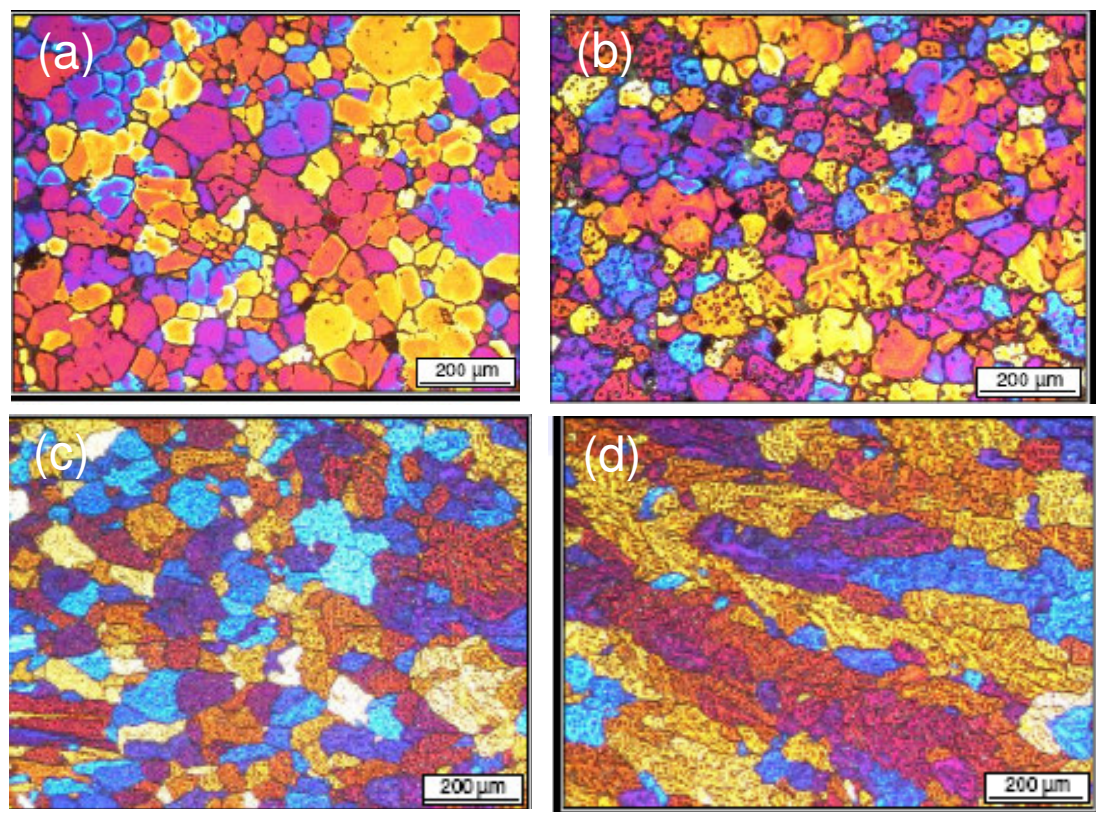

Fig. 2 Microstructures of DC and MC-DC 7075, a) MC-DC, centre b) MC-DC, Edge, c) DC, centre, d) DC, Edge.

The microstructural variations on the cross sections of both DC and MC-DC cast billets were quantified by using an anodising technique to reveal the grain structure and an image analysing facility attached to the optical microscope. Fig. 3 compares the grain size variation of the conventional DC billet with that of the MC-DC billet.

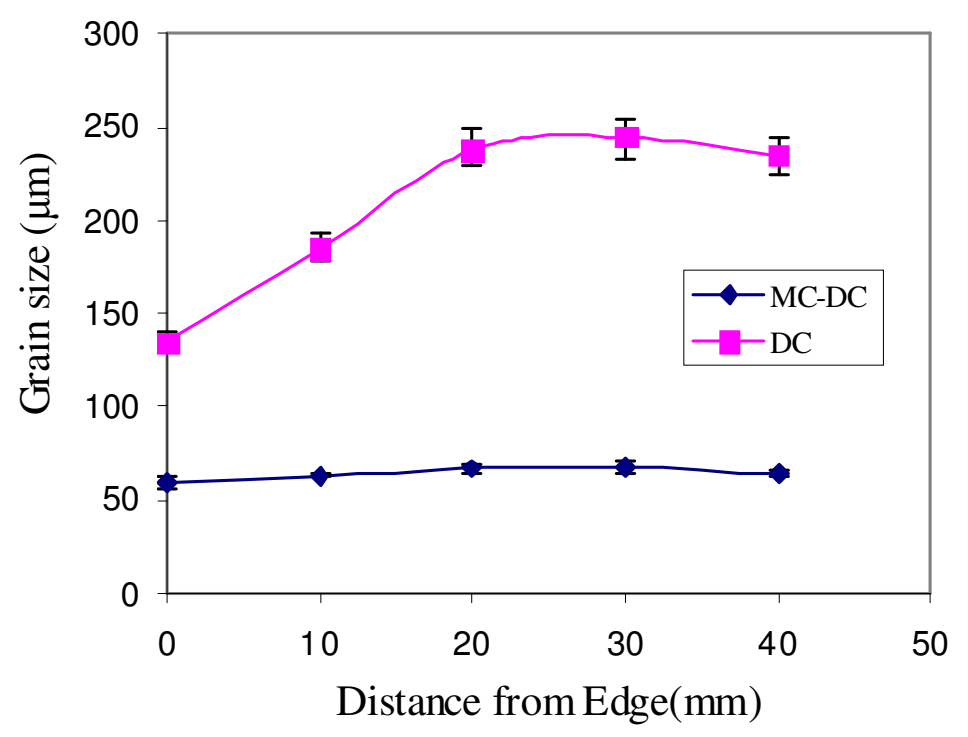

Fig. 3 Grain size variation in MC-DC and DC cast 7075 aluminium alloy.

While the grain size of the DC billet varies from $130 \mu \mathrm{m}$ at the billet surface to about $245 \mu \mathrm{m}$ at the billet centre, the grain size of the MC-DC billet remains fairly constant, being about $60 \mu \mathrm{m}$ throughout the entire cross section. It can be concluded that MC-DC process produces Al-alloy billets which not only have fine grain size but also have homogeneous microstructure throughout the entire billet. One of the challenges in DC casting is to reduce or eliminate macrosegregation in the cast billets. Optical mass spectroscopy was used to quantify the compositional variation of the MC-DC cast 7075 billet. The results are presented in Fig. 4. It is clear from Fig. 4 that the major alloying elements, $\mathrm{Zn}, \mathrm{Mg}$ and $\mathrm{Cu}$, have been distributed uniformly throughout 
the entire cross section of the MC-DC billet, showing no chemical macro-segregations. These results demonstrated that the MC-DC process is capable of producing aluminium alloy billets not only with fine and uniform microstructure but also with homogeneous chemical compositions.

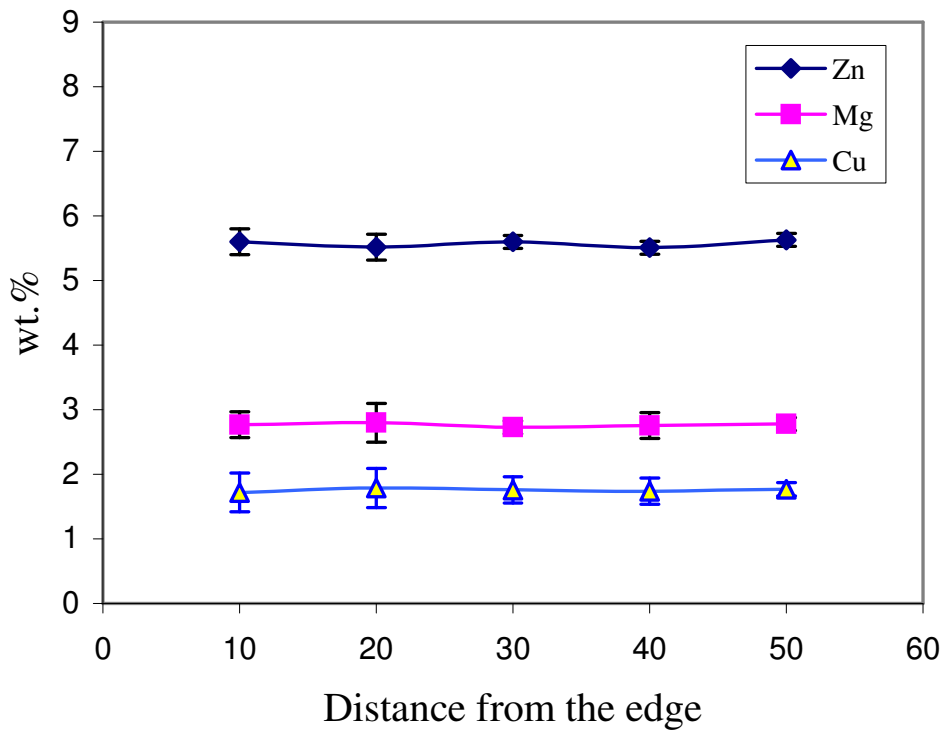

Fig. 4 Chemical composition variation throughout MC-DC 7075 aluminium alloy.

In order to check the interaction between intensive melt shearing and addition of grain refiners, $5754 \mathrm{Al}$-alloy and Al-5Ti-1B grain refiner were selected to conduct DC casting experiments. TP1 mould was used to produce cast sample for microstructural assessment at a specific position, which has a cooling rate of $3.5 \mathrm{~K} / \mathrm{sec}$. The typical microstructures of TP1 samples produced under different conditions are presented in Fig. 5, and the quantified grain sizes of such samples are compared in Fig. 6.

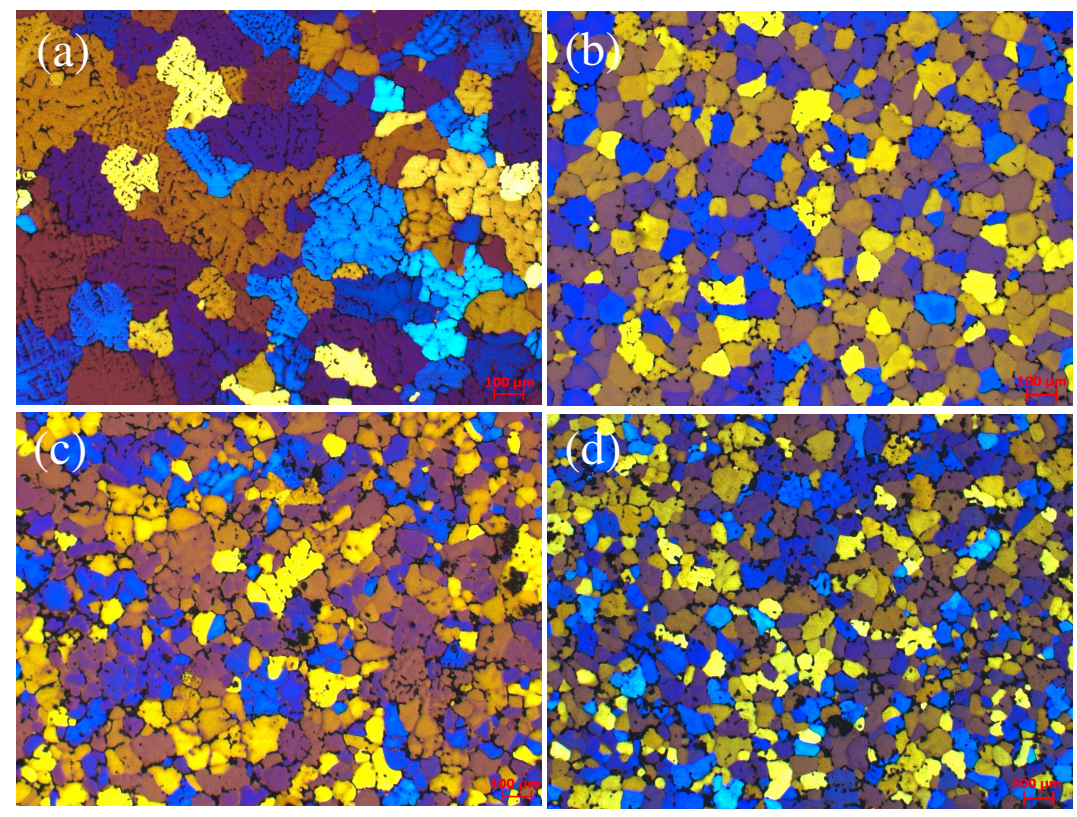

Fig. 5 Microstructural comparison between DC and MC-DC $5754 \mathrm{Al}$ alloy.

(a) Conventional DC casting; (b) DC casting with inoculation; (c) MC-DC; (d) MC-DC with inoculation.

The conventional DC casting without addition of grain refiner and no melt conditioning produces samples with $332 \mu \mathrm{m}$ grain size; $0.1 \%$ addition of Al-5Ti-1B grain refiner can reduce the grain size 
to $124 \mu \mathrm{m}$ while the intensive shearing alone will reduce the grain size to $137 \mu \mathrm{m}$; the combination of grain refiner addition and intensive shearing can reduce the grain size to $98 \mu \mathrm{m}$. It is clear that intensive shearing does make the grain refiner slightly more effective. However, it should be noted that for billets with a grain size in the range of $100-140 \mu \mathrm{m}$ may not have any significant difference in terms of subsequent solid-state deformation processing. It can be concluded that intensive shearing can be used as an effective means for grain refinement to replace the conventional grain refiner.

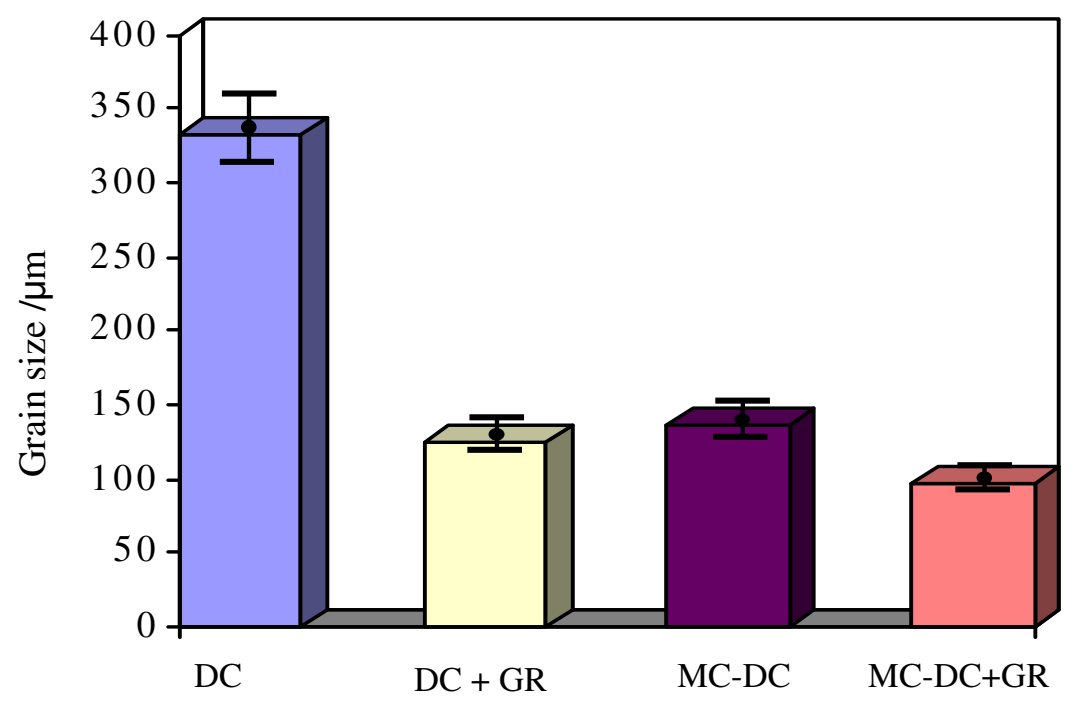

Fig. 6 Comparison of grain size of 5754 aluminium alloy produced under different processing condition, GR refers to grain refiner.

\section{Discussion}

The grain refinement in the MC-DC process can be understood at two different levels. At the macro-scale, the grain refinement can be attributed to the increased effective nucleation rate. The twin screw mechanism in the MCAST machine offers a high shear rate and a high degree of turbulence, as a consequence of which, the temperature and chemical composition of the melt inside the MCAST machine will be extremely uniform. Any heat released by the melt during cooling and solidification will be removed quickly. Such conditions mean that any nuclei created during solidification will survive and contribute to the final solidified microstructure, resulting in substantial grain refinement. This has been named as effective nucleation, in contrast the situation in the conventional casting process, where most of the created nuclei will disappear once they have been transferred to a hotter region [6]. At the micro-level, the enhanced grain refinement can be explained by increasing nucleation potency of the existing oxide particles. Intensive shearing provided by the MCAST machine will convert the oxide particle clusters and oxide films present in the liquid metal into fine and almost micro-sized individual particles due to the excellent dispersive power of the twin-screw mechanism and the Ostwald ripening process under extremely favourable kinetic conditions. According to the free growth model [7], once the appropriate under cooling is achieved, all such oxide particles will act as heterogeneous nucleation sites throughout the entire volume of the conditioned melt. Consequently, it will result in considerable grain refinement. This is again in contrast to the conventional casting processes, where only the large oxide particles (more likely large clusters of oxide particles) will trigger heterogeneous nucleation at very small undercooling and preventing the small oxide particles from being potent for heterogeneous nucleation.

The main advantages of the MC-DC process include fine and uniform microstructure, uniform chemical composition and much reduced cast defects in the final solidified billets or slabs. Other advantages will be increased tolerance to impurities, improve susceptibility to alloy composition, and easy implementation. 


\section{Conclusions}

A novel technology, the MC-DC casting process has been developed by BCAST at Brunel University for production of high quality billets and slabs. This technology is applicable to both Aland Mg-alloys. The main advantages of the MC-DC process include fine and uniform microstructure, uniform chemical composition and much reduced cast defects in the final solidified billets or slabs, as have been demonstrated clearly in this paper by processing 7075 and $5754 \mathrm{Al}-$ alloy. The enhanced microstructural refinement can be attributed to the increased nuclei survival rate and the increased potency of oxide particles by intensive melt shearing.

\section{Acknowledgements:}

The authors would like to thank Dr. G. Scamans, Dr. J. Patel and Mr R. Keyte for their technical help and useful discussions. We would also like to express our gratitude to both EPSRC and DTI (UK) for their financial support.

\section{References:}

[1]. T. Witulski, A.Winkelmann and G. Hirt: $4^{\text {th }}$ Int. Conf. on semisolid processing of alloys and composites, Sheffield (1996), p.242.

[2]. J.P.Gabathuler and D. Barras: $2^{\text {nd }}$ Int. Conf. on semisolid processing of alloys and composites, MIT/Boston (1992), p.33.

[3]. S.M. Zhang, Z. Fan and Z. Zhen: Materials Science and Technology Vol.22 (2006), p.1489.

[4]. Z. Fan, M. Bevis and S. Ji: Patent: PCT/WO 01/21343 A1 (1999).

[5]. "Standard test procedure for aluminium alloy grain refiners (TP-1)", The Aluminium Association, Washington DC, USA (1990).

[6]. Z. Fan and G. Liu: Acta Mater Vol.53 (2005), P.4345.

[7]. A.L.Greer, A.M. Bunn, A. Tronche, P.V. Evans and D.J. Bristow: Acta Materialia Vol.48 (2000), p.2823. 